\title{
A Wireless Body Sensor Network for the Prevention and Management of Asthma
}

\author{
Edmund Y. W. Seto ${ }^{\dagger}$, Annarita Giani ${ }^{\ddagger}$, Victor Shia ${ }^{\ddagger}$, Curtis Wang ${ }^{\ddagger}$, Posu Yan ${ }^{\ddagger}$, Allen Y. Yang ${ }^{\ddagger}$, Michael Jerrett ${ }^{\dagger}$, \\ Ruzena Bajcsy \\ ${ }^{\dagger}$ School of Public Health, University of California, Berkeley, CA 94720 \\ ${ }^{\sharp}$ Department of EECS, University of California, Berkeley, CA 94720
}

\begin{abstract}
We present an application of an open source platform for wireless body sensor network called DexterNet to the problem of children's asthma. The architecture of the system consists of three layers. At the body sensor layer (BSL), the integrated monitoring of a child's activities, geographic location, and air pollution exposures occurs. At the personal network layer (PNL), a wireless mobile device worn by the child summarizes the sensed data, and provides information feedback. The mobile device communicates wirelessly over the Internet with the third global network layer (GNL), in which a web server provides the following four information services: a clinical module that supports the healthcare management of asthma cases, a personal health module that supports individual prevention of asthma attacks, a community module that supports participatory sensing, and a health research module that supports the collection of anonymous sensor data for research into the risk factors associated with asthma. We illustrate the potential for the system to serve as a comprehensive strategy to manage asthma cases and prevent asthma attacks.
\end{abstract}

Keywords: Healthcare services, Asthma, Wireless Sensor Networks, Mobile Computing, Body Sensor Networks

\section{INTRODUCTION}

Asthma is chronic respiratory disease that often starts during childhood, but affects people of all ages. In the U.S. it is estimated that $9.1 \%$ of children (6.7 million) and 16.2 million adults have the disease $[1,2]$. While the causes of asthma are not fully understood, the symptoms of the disease result from a chronic inflammation of the lung's airways that causes episodic periods of wheezing, chest tightness, shortness of breath, and coughing. Intense onsets of severe symptoms - asthma attacks - may require emergency care.

Asthmatics must effectively manage the disease through knowledge and avoidance of the factors that exacerbate symptoms, and the use of medications. Asthma medication can prevent the worsening of symptoms. Another strategy is to avoid common asthma triggers, such allergens (e.g., dust, cockroaches, mold, and pollen), irritants (e.g., cigarette smoke and outdoor air pollution), and exercise. Often triggers are associated with an asthmatic's interaction with the physical environment. For instance, residential proximity to major roads

This work was supported in part by TRUST (The Team for Research in Ubiquitous Secure Technology), which receives support from the National Science Foundation (NSF award number CCF-0424422) and the following organizations: AFOSR (\#FA9550-06-1-0244), Cisco, British Telecom, ESCHER, HP, IBM, iCAST, Intel, Microsoft, ORNL, Pirelli, Qualcomm, Sun, Symantec, Telecom Italia and United Technologies. This work was also supported in part by ARO MURI W911NF-06-1-0076, the Center for Information Technology Research in the Interest of Society (CITRIS). Corresponding author: E. Seto (seto@berkeley.edu). is associated with the presence, severity, and persistence of childhood asthma [3]. Moreover, the relationship between outdoor air pollution and asthma exacerbation [4-7] has led to guidance to reduce physical activity when and where pollution levels are high [8]. However, asthmatics may find it difficult to avoid urban air pollution. Also, avoiding physical activity reduces the many health benefits associated with exercise, and can have social and emotional impacts on children [9].

Technology can play a role in helping asthmatics manage their disease. For example, regional warning programs, like the Bay Area Air Quality Management District's Spare the Air Day program established in 1991 issues public warnings via telephone, email, and mobile phones when air quality forecasts indicate periods of high ozone concentrations so that sensitive populations may limit their time outdoors. However, this regional approach lacks specificity for the asthmatic individual, who may wish to manage their symptoms based on their own activity levels and exposure patterns.

Home telemonitoring, in which an asthmatic are monitored by having clinical and physiologic data transmitted to healthcare providers, is an alternative approach to asthma management. A recent review [10] identified 12 asthma telemonitoring studies, which ranged in sophistication from electronic diaries and spirometer systems to Internet-based monitoring systems. While the systems were generally wellaccepted, adherence was a problem, particularly for complex systems that required difficult transfer procedures for numerous measurements. However, none of studies focused on measuring exposure to environmental triggers for asthma management.

We describe a new telemonitoring system based on a wireless body sensor network (BSN) that may be used as part of a comprehensive approach to managing asthma. The system focuses on monitoring activity patterns and cumulative exposures to environmental air pollution, transmittal of this data to a health information system, and feedback of information to the user on how to manage activity, and reduce the potential for asthma exacerbation. The system leverages the ongoing integration and miniaturization of sensors, processors, and radio technology, and illustrates how existing systems and approaches based on multiple heterogeneous sensors (e.g., CodeBlue [11], HealthGear [12], MobiCare [13], WWBAN [14], ALARM-NET [15], Participatory Sensing [16], and Intel MSP [17]) may be adapted to asthma care. In each of these systems, individuals act as a mobile platform for sensors that monitor the person's normal (indoor-outdoor) living 
environments. Additionally, we describe applications that are built upon our BSN system that illustrate how different perspectives (individual, doctor-patient, community, and health research) may be used collectively for disease management.

\section{A. System Architecture}

The architecture is based on the DexterNet BSN platform [18], a flexible system for heterogeneous wireless BSNs that consists of mote platforms running the TinyOS operating system and an open source software framework for managing on-node sensor data signal processing and between-node communication, arranged in a 3-layer hierarchy (Fig. 1). The bottom layer of the architecture consists of the body sensor layer (BSL), in which one or more TinyOS motes and associated sensors are worn on the body. The motes wirelessly communicate sensor data to a mobile base station at the personal network layer (PNL). A software framework called Signal Processing In Node Environment (SPINE) runs on the motes and base station, allowing data to be processed at the BSL and PNL levels. SPINE also coordinates communication with higher level applications at the global network layer (GNL). These applications can run on the mobile base station device, or alternatively run as web applications that use the base station's wide area network connectivity.

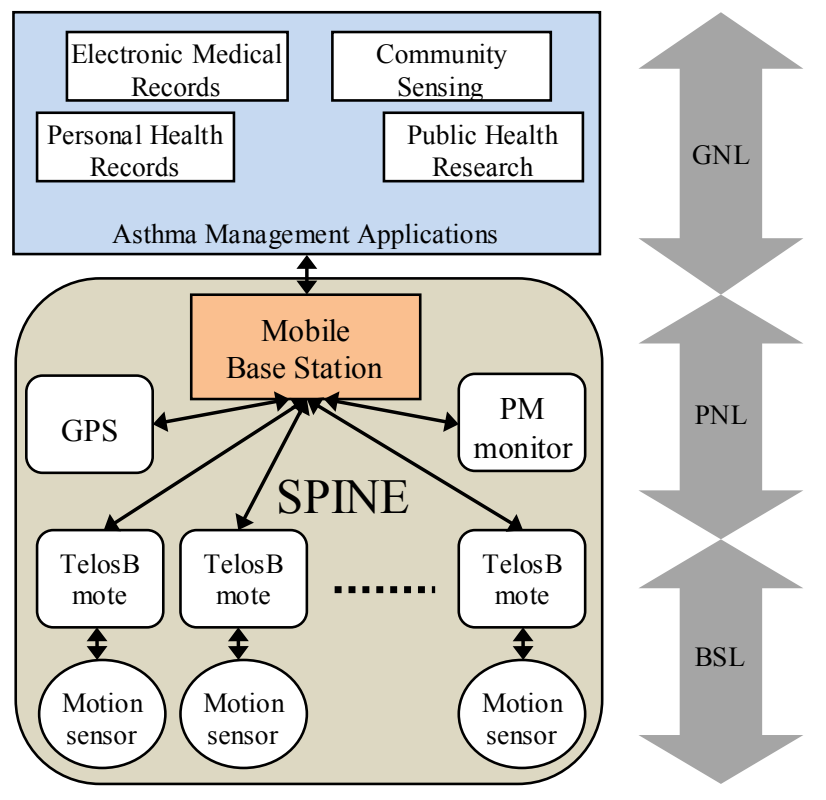

Figure 1. Three-layer architecture of the DexterNet platform for asthma management, consisting of body sensor layer (BSL), personal network layer (PNL), and global network layer (GNL), and integration of TelosB motes and motion sensors, global positioning system (GPS) sensor, and airborne particulate matter (PM) monitor for four asthma management applications.

\section{HARDWARE}

The DexterNet platform was designed at the outset to support a multitude of sensors and TinyOS motes. It also provides flexibility in allowing for the integration of other sensors and monitors that are not TinyOS-based. Below we describe the hardware used in our DexterNet-based asthma system (Fig. 2).

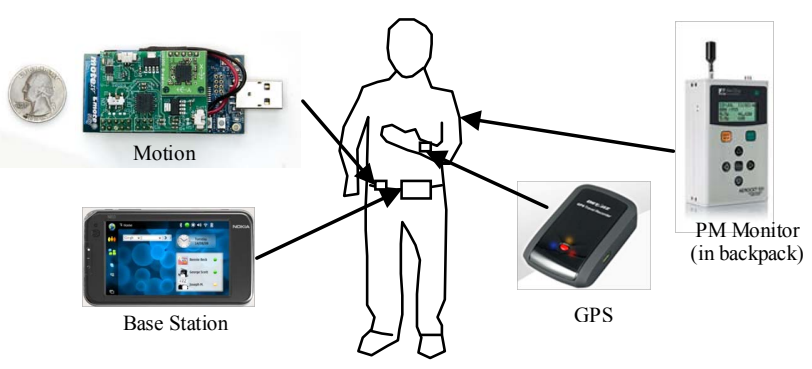

Figure 2. Wireless body sensor network hardware for the asthma management system. Base station consists of Nokia N810 Internet Tablet,

connected wirelessly via 802.15 .4 to custom-built motion sensor mote, wirelessly via Bluetooth to a global positioning system (GPS) receiver, and wired via a Serial-to-USB cable to a airborne particulate matter monitor.

Physical activity is monitored via a custom-built motion sensor board that includes measurements from a triaxial accelerometer and a biaxial gyroscope. The sensor board is attached to a TelosB mote running the TinyOS operating system for wireless network communication and on-node signal processing. The mote communicates using a 802.15 .4 wireless protocol radio and can achieve approximately $64 \mathrm{~Hz}$ maximal sampling rates when raw measurement data are desired. Simple signal processing tasks can be done in real-time with the on-board MSP430F 1611 microcontroller running at 8 $\mathrm{MHz}$ clock frequency with $10 \mathrm{kB}$ of internal RAM. The MSP430 also conducts the conversion of analog measurement signals to digital with 12 bit resolution. The capacity of the battery is $600 \mathrm{mAh}$, which supports maximum continuous measurement and wireless raw data output for approximately 20 hours. An individual may wear one or more motion sensor nodes at different body locations for activity classification and/or physical activity assessment.

A commercially-available Qstarz BT-Q1000P global positioning system (GPS) receiver is used to monitor geographic location of the individual's activity. The receiver is based on the Adopt MTK GPS chipset that provides latitude, longitude, and altitude at a positional accuracy of $3.0 \mathrm{~m} \mathrm{2D}$ RMS. Initial cold start requires 36 seconds to acquire a position fix, after which data are available at 1 -second intervals. The receiver is powered by a lithium ion battery that provides up to 32 hours of operation. The receiver communicates with the base station via a wireless Bluetooth interface.

Air pollution exposure is monitored using a commerciallyavailable Met-One Aerocet 531 Particle Counter. The monitor measures particulate matter (PM), which consists of a wide range of pollutants - dust, soot, fly ash, diesel exhaust particles, wood smoke and sulfate aerosols - that are suspended as airborne particles. PM is commonly characterized by size since fine particles (e.g., $\leq 2.5 \mu \mathrm{m}$ in diameter) reach deep into the lung and are associated with asthma exacerbation. The portable Aerocet optically measures particles via light scattering. The monitor is sensitive to particles $0.5 \mu \mathrm{m}$ to $10 \mu \mathrm{m}$ in size, and provides data as mass concentrations for sizes PM 1, 2.5, 7, and $10 \mu \mathrm{m}$, in addition to total suspended particulates. Samples can be taken manually by the user, or at regular intervals, as often as once every 2 minutes. The unit is powered by a NiCd battery that provides 5 hours of battery life. A RS-232 serial port allows for data communication with the base station. 
A Nokia N810 Internet Tablet serves as the mobile base station at the PNL level. The benefit of the N810 is its USB On-The-Go capability for hosting USB devices, such as the TelosB mote that provides 802.15.4 wireless protocol radio communication with other wireless motes in the system. Additionally, the USB host capability allows it to receive data from the airborne particulate monitor via a wired serial-to-USB interface. The N810 also has Bluetooth capability that allows it to receive wireless data from the GPS. Finally, the device can communicate with the GNL via its onboard $802.11 \mathrm{Wi}-\mathrm{Fi}$ radio or alternatively via Bluetooth to a mobile phone modem. The N810 has 6 hours of battery life under typical usage, and has an onboard keyboard and touch screen that can support user input and feedback. The N810 runs the Maemo operating system, an open source version of Linux, and supports application programming in both $\mathrm{C}$ and Java languages. Applications can use the device's $2 \mathrm{~GB}$ of expandable memory for data logging.

\section{SPINE FRAMEWORK}

The system uses Signal Processing In Node Environment (SPINE), an open-source framework for distributed signal processing algorithms in wireless sensor networks (WSNs). SPINE operates primarily in the BSL and PNL layers, providing a set of on-node services written in TinyOS for sensing, signal processing, and communication, and a set of corresponding base station services written in Java. SPINE greatly facilitates the design of WSN applications through abstraction of sensing, signal processing, and communication components, allowing application developers to use preexisting sensor data processing algorithms, and programming in Java on the base station, while retaining the flexibility to allow more sophisticated on-node data processing to be implemented in TinyOS as needed. Additionally, SPINE provides an efficient wireless communication protocol for dynamic network configuration and management (e.g., the ability to turn on/off or reconfigure collection, processing, and transmission of data from particular sensors at runtime). The benefits of SPINE's communication architecture are described in detail elsewhere [29].

The system uses of an on-node energy expenditure algorithm called KCal implemented in SPINE. The KCal algorithm is based on on-node integration of the vertical and horizontal components of the accelerometry data measured at the person's hip, and a generalized linear model based on mass, height, and age to estimate energy expenditure in KJ per minute [19].

Because the air pollution and GPS data are provided by commercial devices rather than TinyOS-based motes, technically, they are outside of the SPINE framework. However, Java functions that access USB/serial data can be called by SPINE on the base station. When GPS data are available, GPSD - a daemon that runs as a service on the N180 -calls a Java function to pass the data to the SPINE application. For air pollution data, SPINE Java code directly accesses the serial port on the N810 and parses the air pollution data that are sent by the Aerocet after each air sample is analyzed. This "loose" integration acknowledges the need to leverage available sensors and proprietary devices in our system.

\section{Global Network LAyer Web APPlicAtions}

The combined motion, location, and PM concentration data in the SPINE Java application on the N810 can form the basis of a comprehensive asthma management telemonitoring system. To support such a system, we implemented a webservice (Apache webserver, MySQL database, and PHP scripts to process HTTP Post requests) that receives this information at regular intervals from the base station over an Internet connection.

This can support a Personal Health Record web application, in which asthmatics manage their own disease by viewing their air pollution exposure patterns to identify periods of high exposure in relationship to their own experience with symptoms and dosing of medication so that they can effective plan reduced activity and exposure (Fig. 3).

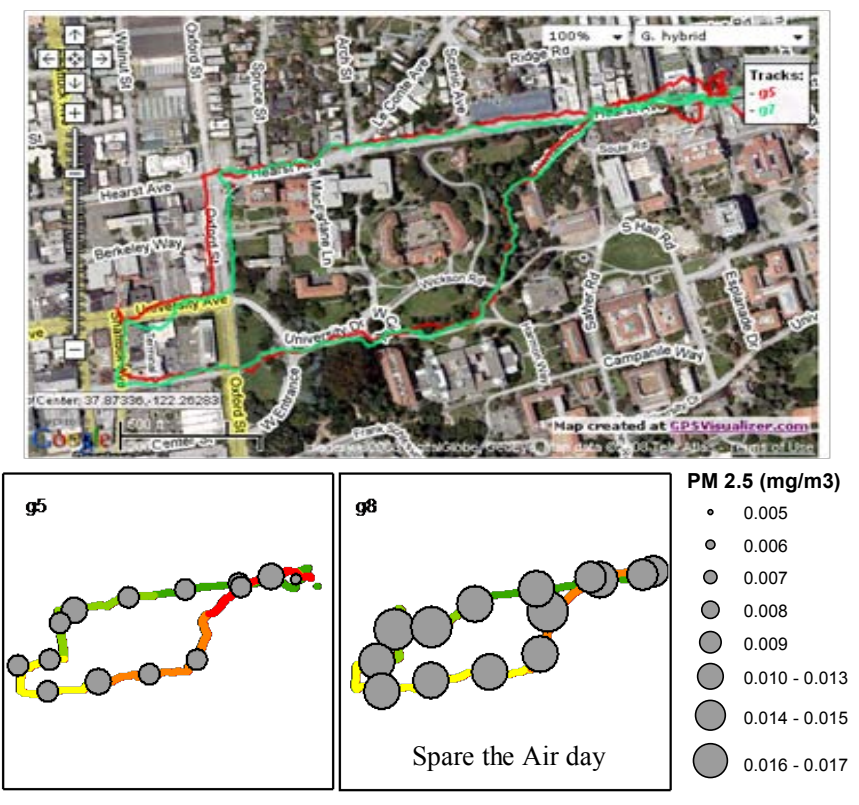

Figure 3. Illustration of two person's activity patterns with the system on separate days, one a Spare the Air day with higher PM 2.5 concentrations.

Another web application is an Electronic Medical Record System, which focuses on a healthcare provider's need to monitor patients. Health providers monitor patients' exposure data to inform treatment decisions. Continuous and near realtime data may lead to new decision-support tools aimed at notifying patients (e.g., when cumulative exposures exceed a threshold), and comparison between patients to identify treatments that are likely to succeed for similar populations.

A third application is Participatory Sensing, which is a community approach to reducing environmental risks. For this, many asthmatics collectively contribute air pollution data to the database. These data are likely to be at a finer spatial and temporal resolution than regulatory fixed site monitors. Maps of interpolated concentrations may help city, transportation, and health planners improve urban design to mitigate air pollution hotspots and reduce environmental justice issues.

Finally, a fourth application is a Health Research System, in which epidemiologists can access aggregated and nonpersonally identifiable data to better inform the relationships 
between environmental exposure and asthma exacerbation, which thus far has largely relied upon residential location as a proxy for true exposure.

Together, these four components offer a comprehensive set of individual, doctor-patient, community, and health research tools for asthma management that focuses on physical activity and environmental triggers that exacerbate symptoms.

\section{DISCUSSION}

While we have demonstrated the feasibility of a comprehensive wireless BSN for asthma, the realization of these systems must solve several real-world challenges. Sensor data that describe an individual's activity patterns and exposures are extremely personal, and require various safeguards to prevent inadvertent disclosure. Permission schemes may be useful in allowing users to choose whether to disclose data. If personal data are considered health-related, in the U.S., Health Insurance Portability and Accountability Act (HIPAA) privacy assurances apply. Also, data encryption and authentication are needed throughout the BSL to GNL levels.

Another challenge is the integration of mobile sensors, which in our demonstration consists of an arguably bulky setup. But, there is ongoing work within our group and others to better integrate improved air quality sensors and battery life for health research. Addition of a physiologic sensor that measure respiration, which was included in our group's earlier DexterNet work may be an improvement [18]. But, complex, invasive, or too many sensors may impact user acceptance.

We have addressed some of the challenges in previous respiratory health telemonitoring studies with our system that transmits data from an integrated set of sensors that tracks activity and exposures with very little user interaction. However, cost-effectiveness remains to be considered both via evaluation of efficacy in a rigorous controlled study and whether the costs of such a system are warranted. Hardware costs can likely be reduced through integration, however, human resource costs must be considered, particularly for EMR applications that require healthcare providers monitor and provide a response to near real-time data. Ultimately, automated systems to triage data and integrate them into a set of efficient and standard decision protocols are needed.

Nevertheless, because there are many asthmatics and huge economic costs associated with the disease, even low levels of efficacy may make a telemonitoring system worthwhile. In the U.S. alone, 10.6 million physician office visits and 444,000 hospital visits related to asthma [20,21], and estimated asthmarelated costs of $\$ 6.2$ billion (US dollars in 1990) [22] motivate the need for continued research into innovative approaches to managing asthma.

\section{ACKNOWLEDGMENTS}

We thank Dr. Marco Sgroi, Roberta Giannantonio, and Raffaele Gravina at the WSN Lab Berkeley; Kartherine Gilani and Dr. Roozbeh Jafari at the University of Texas Dallas; Philip Kuryloski and Stephen Wicker at Cornell University; and Ville-Pekka Seppä and Jari Hyttinen at Tampere University of Technology.

\section{REFERENCES}

[1] J. R. Pleis and J. W. Lucas, "Summary health statistics for U.S. adults: National Health Interview Survey, 2007," National Center for Health Statistics. Vital Health Stat, 2009.

[2] B. Bloom and R. A. Cohen, "Summary health statistics for U.S. children: National Health Interview Survey, 2007," National Center for Health Statistics. Vital Health Stat, 2009.

[3] R. McConnell, K. Berhane, L. Yao, M. Jerrett, F. Lurmann, F. Gilliland, et al., "Traffic, susceptibility, and childhood asthma," Environ Health Perspect, vol. 114, pp. 766-72, May 2006.

[4] R. J. Delfino, R. S. Zeiger, J. M. Seltzer, and D. H. Street, "Symptoms in pediatric asthmatics and air pollution: differences in effects by symptom severity, anti-inflammatory medication use and particulate averaging time," Environ Health Perspect, vol. 106, pp. 751-61, Nov 1998.

[5] K. M. Mortimer, L. M. Neas, D. W. Dockery, S. Redline, and I. B. Tager, "The effect of air pollution on inner-city children with asthma," Eur Respir J, vol. 19, pp. 699-705, Apr 2002.

[6] B. Ostro, M. Lipsett, J. Mann, H. Braxton-Owens, and M. White, "Air pollution and exacerbation of asthma in African-American children in Los Angeles," Epidemiology, vol. 12, pp. 200-8, Mar 2001.

[7] HEI Panel on the Health Effects of Traffic-Related Air Pollution, "Traffic-Related Air Pollution: A Critical Review of the Literature on Emissions, Exposure, and Health Effects," Special Report 17, 2009.

[8] U.S. Environmental Protection Agency, "Asthma and outdoor air pollution," EPA-452-F-04-002, 2009.

[9] A. Nocon, "Social and emotional impact of childhood asthma," Arch Dis Child, vol. 66, pp. 458-60, Apr 1991.

[10] M. Jaana, G. Pare, and C. Sicotte, "Home telemonitoring for respiratory conditions: a systematic review," Am J Manag Care, vol. 15, pp. 313-20, May 2009.

[11] D. Malan, T. Fulford-Jones, M. Welsh, and S. Moulton, "CodeBlue: An ad hoc sensor network infrastructure for emergency medical care," In Workshop on Wearable and Implantable Body Sensor Networks, 2004.

[12] N. Oliver and F. Flores-Mangas, "HealthGear: A real-time wearable system for monitoring and analyzing physiological signals," In Workshop on Wearable and Implantable Body Sensor Networks, pp. 6164, 2006.

[13] R. Chakravorty, "A programmable service architecture for mobile medical care," In Pervasive Comp. and Comm. Workshop, 2006.

[14] A. Milenkovic, C. Otto, and E. Jovanov, "Wireless sensor networks for personal health monitoring: Issues and an implementation," Computer Communications, vol. 29, pp. 2521-2533, 2006.

[15] A. Wood, G. Virone, T. Doan, Q. Cao, L. Selavo, Y. Wu, et al., "ALARM-NET: Wireless sensor networks for assisted-living and residential monitoring," Technical report, Department of Computer Science, University of Virginia, 2006.

[16] J. Burke, D. Estrin, M. Hansen, A. Parker, N. Ramanathan, S. Reddy, et al., "Participatory sensing," In World Sensor Web Workshop, 2006.

[17] T. Choudhury, S. Concolvo, B. Harrison, J. Hightower, A. LaMarca, L. LeGrand, et al., "The mobile sensing platform: An embedded activity recognition system," Pervasive Computing, pp. 32-41, 2008.

[18] P. Kuryloski, A. Giani, R. Giannantonio, K. Gilani, R. Gravina, V. Seppä, et al., "DexterNet: An Open Platform for Heterogeneous Body Sensor Networks and Its Applications," BSN 2009, 2009.

[19] K. Y. Chen and M. Sun, "Improving energy expenditure estimation by using a triaxial accelerometer," J Appl Physiol, vol. 83, pp. 2112-22, Dec 1997.

[20] D. K. Cherry, E. Hing, D. A. Woodwell, and E. A. Rechtsteiner, "National Ambulatory Medical Care Survey: 2006 Summary " National Health Statistics Reports, vol. 3, 2008.

[21] C. J. DeFrances, C. A. Lucas, V. C. Buie, and A. Golosinskiy, "2006 National Hospital Discharge Survey " National Health Statistics Reports, vol. 5, 2008.

[22] K. B. Weiss, P. J. Gergen, and T. A. Hodgson, "An economic evaluation of asthma in the United States," N Engl J Med, vol. 326, pp. 862-6, Mar 261992. 